\title{
Predictores de síndrome coronario agudo sin supradesnivel del ST y estratificación de riesgo en la unidad de dolor torácico. Experiencia en 1.168 pacientes
}

\author{
Luigi A Gabrielli, Pablo F Castro, Hugo E Verdejo, \\ Paul A McNab, Silvana A Llevaneras, José M Mardonez, \\ Ramón L Corbalán \\ Predictors of acute coronary \\ syndrome without ST segment \\ elevation and risk stratification \\ in the chest pain unit
}

Background: Nearly $10 \%$ of patients with an actual acute coronary syndrome (ACS) are discharged with an inadequate diagnosis. Aim: To select clinical and laboratory predictors to identify patients with a high likelihood of ACS in the Chest Pain Unit. Material and methods: Prospective evaluation of patients consulting in a Chest Pain Unit of a University Hospital. Initial assessment was standardized and included evaluation of pain characteristics, electrocardiogram and Troponin I. Independent predictors of ACS were identified with a multiple logistic regression. Results: In a four years period, 1,168 patients aged $62 \pm 23$ years ( $69 \%$ males), were studied. After initial evaluation, $62 \%$ of the patients were admitted to the hospital for further testing and in $71 \%$ of them, a definite diagnosis of ACS was made. No events were reported by patients directly discharged from the Chest Pain Unit. Independent predictors associated with a higher likelihood of ACS were an abnormal electrocardiogram at the initial evaluation (Odds ratio (OR) 5.37, 95\% confidence intervals (CI) 3.61-7.99), two or more cardiovascular risk factors (OR 2.16, 95\% CI 1.21-2.84), cervical irradiation of the pain (OR $1.84,95 \%$ CI 1.25-2.69), age over 65 years (OR 1.73, 95\% CI (1.32-2.27) and a Troponin I above the upper normal limit (OR: 5.68, 95\% CI 3.72-8.29). Conclusions: Simple clinical findings allow an appropriate identification of patients with a high likelihood of ACS without specialized methods for myocardial ischemia detection (Rev Méd Chile 2008; 136: 442-50).

(Key words: Acute coronary syndrome; Risk factors; Troponin T-1)

Recibido el 27 de julio, 2007. Aceptado el 13 de noviembre, 2007.

Departamento de Enfermedades Cardiovasculares, Pontificia Universidad Católica de Chile. Santiago de Chile.

Correspondencia a: Dr. Pablo Castro G. Marcoleta 367, $6^{\circ}$

piso. Teléfono: 3543334 . Fax: 6392037.

E mail: pcastro@med.puc.cl 
$\mathrm{L}^{\mathrm{a}}$ a consulta por dolor torácico (DT) representa entre 5\% y 20\% de las consultas al servicio de urgencia (SU) en los hospitales generales ${ }^{1} \mathrm{y}$ constituye un importante desafío diagnóstico por la severidad de algunas de las potenciales etiologías, siendo la situación diagnóstica más compleja la sospecha de un síndrome coronario agudo (SCA) sin supradesnivel del segmento ST. Una evaluación expedita y acuciosa es fundamental para confirmar o descartar un SCA; sin embargo, 60\% de las admisiones por sospecha de SCA corresponden a etiología no coronaria ${ }^{1,2}$. Paralelamente, entre $2 \%$ y $10 \%$ de los pacientes con DT que son dados de alta desde el SU con diagnóstico de patología osteomuscular están cursando con un infarto agudo al miocardio, con una mortalidad que se duplica en relación a los enfermos ingresados en su primera evaluación ${ }^{3}$. Errores de este tipo constituyen entre $20 \%$ y 39\% de los motivos de demandas judiciales en Estados Unidos de Norteamérica $^{4}$. La evaluación clínica y electrocardiográfica inicial constituyen las principales herramientas para identificar a los sujetos con alta probabilidad de SCA entre los pacientes que concurren por DT y es con estos elementos con los cuales debe tomarse la decisión del ingreso o control ambulatorio de estos pacientes 5 . Aun cuando algunos estudios han establecido características sintomáticas predictoras de SCA, éstas carecen de la sensibilidad necesaria para la toma de una decisión definitiva y sus conclusiones son discordantes $^{6}$. En la gran mayoría de los pacientes que consultan por DT indiferenciado existen, además, alteraciones electrocardiográficas inespecíficas cuyo significado diagnóstico no es claro ${ }^{5}$, lo que contribuye a dificultar la toma de decisiones. Por otro lado, si bien la estratificación de riesgo en los pacientes con SCA ya diagnosticado está bien establecida ${ }^{7-11}$, en los pacientes que consultan por DT, el valor predictivo y los resultados de la evaluación clínica, electrocardiográfica y de laboratorio integrada no está bien establecida, en especial considerando la no utilización de métodos especializados de detección de isquemia.

Para mejorar la efectividad en el diagnóstico del dolor torácico y prevenir altas erróneas se han propuesto varias medidas, entre ellas, la atención de estos pacientes en áreas específicas ${ }^{12-14}$. Esta última solución se conoce con el nombre de
Unidad de Dolor Torácico (UDT). Actualmente, disponemos de los resultados del funcionamiento de estas UDT de centros hospitalarios en diferentes lugares del mundo ${ }^{15-17}$.

En agosto de 2002 se creó nuestra UDT, con el objeto de mejorar la atención del DT en un centro terciario que atiende alrededor de 10.000 consultas de urgencias anuales. Desde su implementación, y a diferencia de otras UDT, las decisiones clínicas se han realizado prescindiendo de la utilización de pruebas de provocación o imágenes de detección de isquemia.

El objetivo del presente trabajo es determinar las características clínicas y electrocardiográficas que permiten identificar a los sujetos con alta probabilidad de SCA sin supradesnivel del segmento ST entre los pacientes que acuden a un SU con DT y mostrar los resultados de una UDT que no utiliza pruebas de isquemia en la evaluación inicial.

\section{PACIENTES Y MÉTODOS}

Estudio prospectivo de pacientes que concurrieron por DT no traumático al SU del Hospital Clínico de la Pontificia Universidad Católica de Chile en horario diurno, en el período comprendido entre agosto de 2002 y julio de 2006. La UDT fue organizada dentro del propio SU. El personal a cargo incluía un médico en formación como especialista en cardiología junto a un cardiólogo de la planta, apoyado por enfermeras y auxiliares de enfermería entrenadas en el reconocimiento oportuno del dolor coronario, interpretación básica del electrocardiograma (ECG) y fundamentos de terapia y reanimación cardiovascular. Se registraron antecedentes clínicos, características semiológicas del dolor y alteraciones electrocardiográficas. En pacientes con probabilidad intermedia o alta de SCA se solicitó troponina I al momento de ingreso y luego de 6 h, según protocolo (Figura 1). No se utilizaron pruebas de provocación de isquemia.

Luego de la evaluación inicial y estratificación de riesgo del paciente, se procedió a una de las siguientes conductas: hospitalización, egreso al domicilio u observación en la UDT, según lo determinara el médico responsable del paciente.

En todos los pacientes hospitalizados se registró el diagnóstico definitivo al alta. En los pacien- 


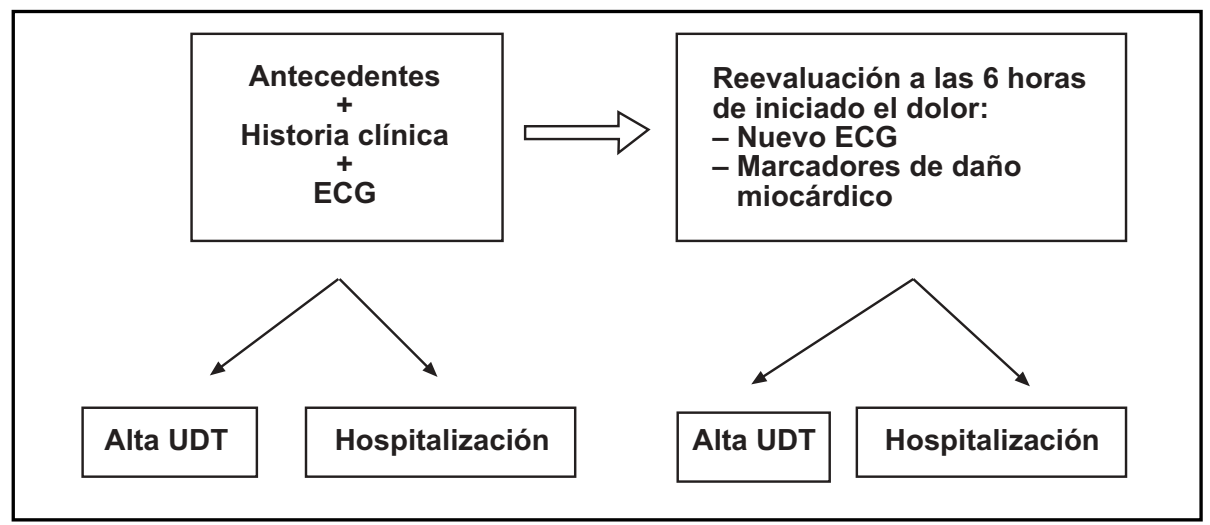

Figura 1. Protocolo de actuación ante un dolor torácico sospechoso de SCA.

tes enviados a su domicilio directamente desde el SU o luego del período de observación, se realizó un seguimiento telefónico a los 15 días, consignándose nuevas consultas a otros $\mathrm{SU}$, hospitalización por SCA o mortalidad.

Dentro del diagnóstico de SCA se incluyó a pacientes con infarto agudo de miocardio (IAM) sin supradesnivel del segmento ST (definido por la presencia de troponina $\mathrm{I}>0,5 \mathrm{mg} / \mathrm{ml}$ ) y angor inestable (definido por la presencia de dolor torácico sugerente y alteraciones electrocardiográficas sugerentes en ausencia de elevación enzimática). Los pacientes que ingresaron con diagnóstico de IAM con supradesnivel ST fueron excluidos del análisis.

Dentro de la evaluación clínica inicial se definió "electrocardiograma anormal" como la presencia de una o más de las siguientes alteraciones en el electrocardiograma de ingreso: 1 . Supradesnivel del segmento ST entre 0,5 y $1 \mathrm{~mm}$ en al menos 2 derivaciones contiguas; 2 . Infradesnivel del segmento $\mathrm{ST} \geq 1 \mathrm{~mm}$ en al menos dos derivaciones contiguas; 3 . Inversión de la onda $T$ en 3 o más derivaciones; 4. Bloqueo completo de rama izquierda o derecha y 5 . Presencia de QS en 2 o más derivaciones. Los niveles de troponina I (ensayo AccuTnI de Beckman Coulter tm) se interpretaron según los límites fijados por el laboratorio local. Un nivel plasmático superior a $0,05 \mathrm{mg} / \mathrm{ml}$ fue considerado como positivo.

Las variables predictoras univariadas de SCA se incluyeron en un modelo de regresión logística múltiple escalonada para identificar los predictores independientes, ajustados por edad y sexo de
SCA. Para los pacientes con el diagnóstico de infarto sin supradesnivel ST, definido como la concomitancia de signos clínicos, electrocardiográficos o ambos, característicos de isquemia asociados a elevación de biomarcadores de necrosis miocárdica (troponina I, CKMB o ambos), se construyó un puntaje predictivo con los elementos clínicos y electrocardiográficos de la evaluación inicial. Para la elaboración de este puntaje se utilizaron los coeficientes de la regresión logística obtenidos en una muestra aleatoria de $75 \%$ de la población en estudio. El restante $25 \%$ fue empleado para la validación de dicho puntaje. Todos los análisis se realizaron con el software SPSS 12.0, Illinois, MA USA.

\section{Resultados}

Entre agosto de 2002 y julio de 2006 fueron evaluados en la UDT de nuestro hospital 1.168 pacientes, correspondiendo a $4,8 \%$ de la totalidad de las consultas en el SU. Las características demográficas se muestran en la Tabla 1 . La edad promedio de los pacientes fue de $62 \pm 23$ años y $69 \%$ eran hombres. La duración media del dolor antes de la consulta fue de $240 \pm 64 \min$ y en $15 \%$ de los pacientes presentó alguna irradiación característica (Tabla 2). El 33,1\% de los pacientes presentaba un ECG normal al momento de la consulta (Tabla 3).

Luego de la evaluación inicial, $62 \%$ de los pacientes fue hospitalizado directamente, $20 \%$ fue 
Tabla 1. Antecedentes clínicos

\begin{tabular}{|lr|}
\hline Número de pacientes & 1.168 \\
Período & agosto de 2002-julio de 2006 \\
Edad (años) \pm DE & $62 \pm 23$ \\
Sexo masculino & $69 \%$ \\
Hipertensión arterial & $49 \%$ \\
Diabetes mellitus & $15 \%$ \\
Dislipidemia & $39 \%$ \\
Tabaquismo & $33 \%$ \\
Presencia $\geq 2$ FRCV & $68 \%$ \\
\hline
\end{tabular}

FRCV: factor de riesgo cardiovascular

Tabla 2. Características semiológicas asociadas al dolor

\begin{tabular}{|lr|}
\hline Duración del dolor (min) \pm DE & $240 \pm 64$ \\
Irradiación al cuello & $17 \%$ \\
Irradiación al dorso & $15 \%$ \\
Irradiación al brazo derecho & $8 \%$ \\
Irradiación al brazo izquierdo & $28 \%$ \\
Irradiación a la mandíbula & $9 \%$ \\
Disnea & $7 \%$ \\
Síncope & $0,6 \%$ \\
Náusea & $9 \%$ \\
Diaforesis & $18 \%$ \\
\hline
\end{tabular}

observado en la UDT y 18\% fue enviado a su domicilio. En los pacientes observados en la UDT, el tiempo promedio fue de $134 \pm 64 \mathrm{~min}$. La conducta final en estos pacientes fue la hospitalización en $15 \%$ de los casos y el egreso a su domicilio en $85 \%$. El diagnóstico de egreso de los pacientes dados de alta desde la UDT se especifica en la Tabla 4. Del total de pacientes hospitalizados, se confirmó el diagnóstico de SCA en 71\% de los casos. El seguimiento telefónico a los 15 días se efectuó en $95 \%$ de los pacientes enviados a su domicilio, sin que se registraran eventos cardiovasculares o muerte en alguno de ellos (Figura 2).

Las variables asociadas a una mayor probabilidad de SCA fueron la presencia de un ECG anormal al momento de la consulta (OR: 5,37 IC 95\% 3,61-7,99), el antecedente de más de un factor de riesgo cardiovascular (OR: 2,16 IC 954\% 1,21-2,84), la irradiación cervical del dolor toráci-
Tabla 3. C aracterísticas electrocardiográficas

\begin{tabular}{|lr|}
\hline Electrocardiograma normal & $33,10 \%$ \\
Supradesnivel ST $\leq 1 \mathrm{~mm}$ & $8,27 \%$ \\
Infradesnivel ST $\geq 1 \mathrm{~mm}$ & $9,47 \%$ \\
Inversión onda T & $16,80 \%$ \\
Presencia de QS & $8,44 \%$ \\
BCRD & $4,48 \%$ \\
BCRI & $2,15 \%$ \\
\hline
\end{tabular}

BCRD: bloqueo completo rama derecha. BCRI: bloqueo completo rama izquierda.
CO (OR: 1,84 IC 95\% 1,25-2,69) y una edad mayor de 65 años (OR 1,73 IC 95\% 1,32-2,27) (Figura 3). La presencia de un ECG anormal al momento de la consulta tuvo un valor predictivo positivo de $40 \%$ para SCA y un ECG normal tuvo un valor predictivo negativo de $91 \%$. De los pacientes

Tabla 4. D iagnóstico pacientes dados de alta directamente desde la UDT

\begin{tabular}{|lr|}
\hline Dolor osteomuscular & $38 \%$ \\
Pleura-pericárdico & $12 \%$ \\
Esofagitis/RGE & $11 \%$ \\
Espasmo esofágico & $6 \%$ \\
Crisis de pánico/ansiedad & $17 \%$ \\
Otros & $15 \%$ \\
\hline
\end{tabular}

RGE: reflujo gastroesofágico. 


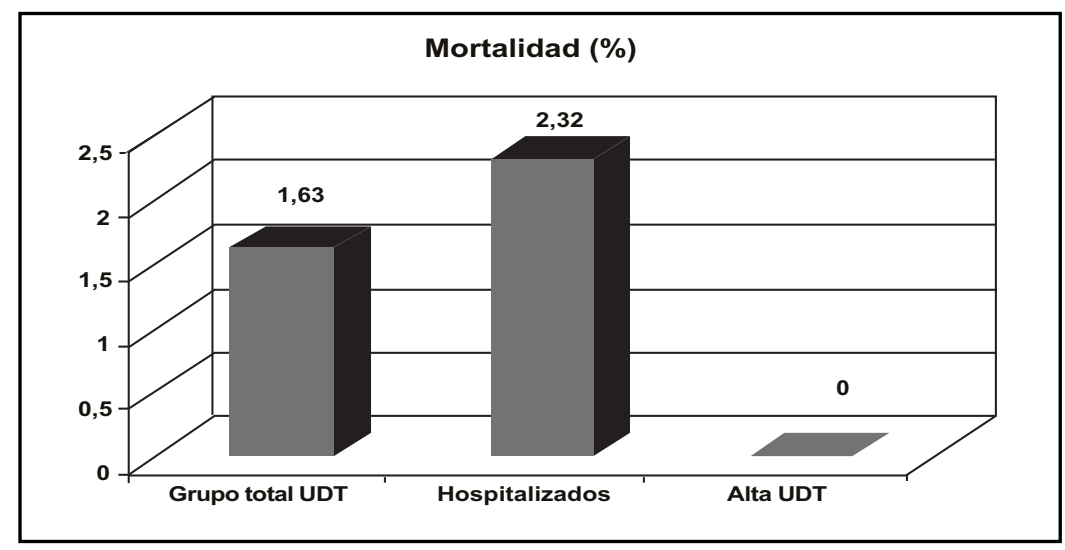

Figura 2. Mortalidad en el seguimiento a 15 días.

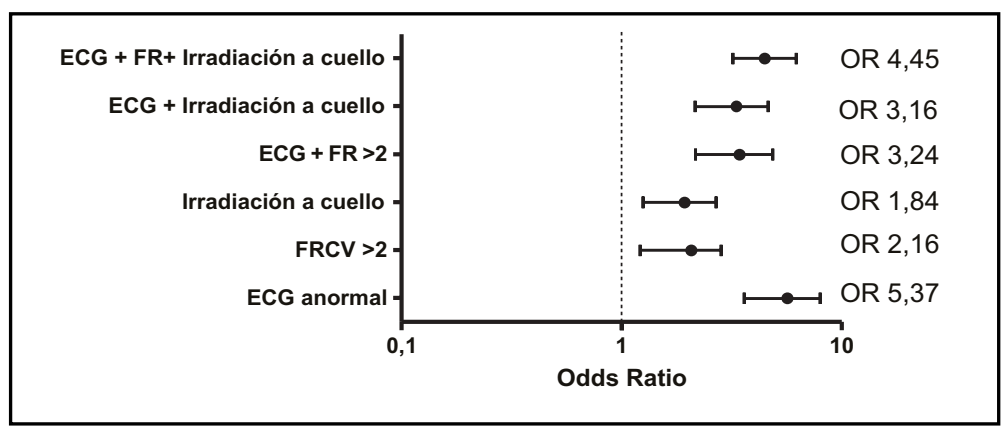

Figura 3. Predictores independientes de SCA.

hospitalizados, 80\% tenía un ECG anormal comparado con sólo 36\% de los pacientes dados de alta desde la UDT (Figura 4). Dentro de las alteraciones electrocardiográficas, excluyendo el supradesnivel del segmento ST $>1 \mathrm{~mm}$, fueron predictoras para SCA el supradesnivel del ST entre 0,5 y $1 \mathrm{~mm}$ y el infradesnivel del segmento ST $>1 \mathrm{~mm}$ (Figura 5). Excluyendo las variables clínicas y electrocardiográficas, la presencia de troponina I elevada $(>0,05 \mathrm{mg} / \mathrm{ml})$ constituye, como es evidente, un poderoso marcador de SCA en pacientes que consultan por dolor torácico OR: 5,68 (3,72-8,29).

La integración de las variables clínicas y electrocardiográficas permitió la construcción de un puntaje diagnóstico para infarto sin supradesnivel del ST (Tabla 5). Considerando un corte de 8 puntos en el puntaje se tiene una sensibilidad y especificidad de $86 \%$ y $72 \%$, respectivamente; los distintos valores predictivos positivos se muestran en la Tabla 5. La ausencia de puntaje tiene un valor predictivo negativo de 96\% para el diagnóstico final de infarto sin supradesnivel del ST, como se definió anteriormente.

\section{DisCUSIÓN}

La relevancia del DT como motivo de consulta en los SU justifica contar con protocolos que optimicen los recursos disponibles, minimizando el riesgo de altas inadecuadas. Con este fin, el desarrollo de aproximaciones estandarizadas basadas en una evaluación clínica y de laboratorio sistemática de los pacientes que consultan por dolor torácico parece ser un enfoque adecuado ${ }^{18}$.

Datos provenientes de estudios observacionales han demostrado las limitaciones de la anamnesis para identificar pacientes con SCA. En efecto, en un seguimiento en la cohorte de Framingham, cerca 


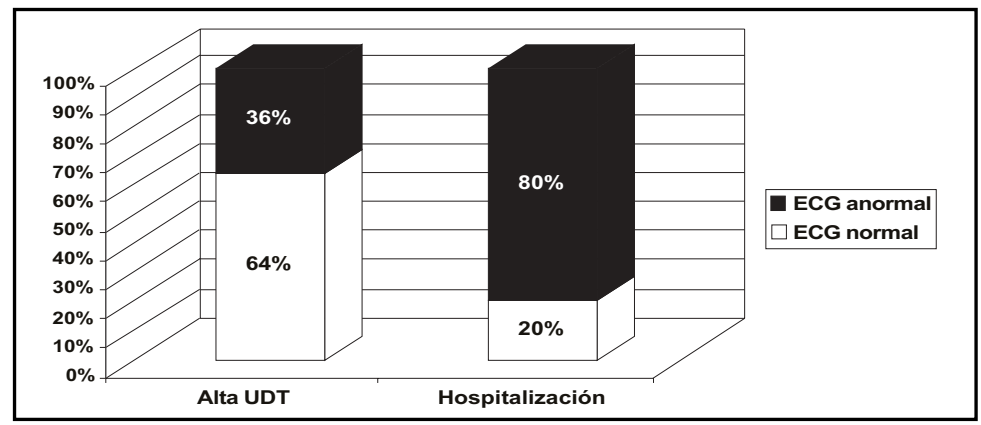

Figura 4. Presencia de ECG normal y anormal en pacientes hospitalizados y dados de alta desde la UDT.

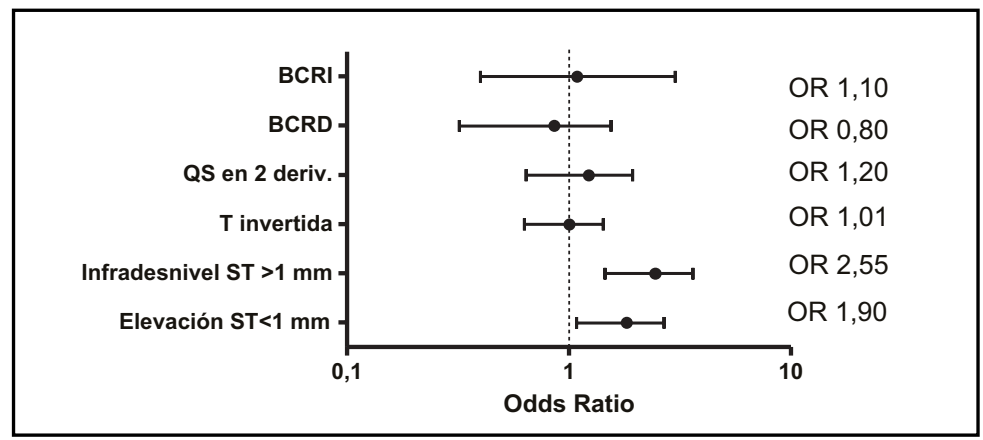

Figura 5. Predictores electrocardiográficos de SCA.

Tabla 5. Puntaje diagnóstico para infarto sin supradesnivel del segmento ST en la evaluación inicial de la UDT

\begin{tabular}{|lc|}
\hline$>65$ años & 2 puntos \\
Irradiación cervical del dolor & 2 puntos \\
$>2$ FRCV & 3 puntos \\
ECG anormal & 5 puntos \\
\hline Puntaje & Diagnóstico final infarto \\
& sin SDST \\
\hline 2 & $45 \%$ \\
$3-4$ & $46-57 \%$ \\
$5-7$ & $58-67 \%$ \\
$8-9$ & $68-75 \%$ \\
$10-12$ & $76-82 \%$ \\
\hline
\end{tabular}

SDST: supradesnivel del segmento ST.

de $25 \%$ de los infartos no fueron reconocidos en la primera consulta, por ausencia de dolor o presencia de síntomas atípicos ${ }^{19}$. Aun cuando en nuestra serie algunas características clínicas como la edad sobre 65 años, la presencia de factores de riesgo y la irradiación típica del dolor constituían fuertes elementos diagnósticos, la variabilidad de los datos obtenidos en la historia clínica constituye un factor a considerar, tal como muestra un estudio publicado por Hickan y cols, donde los elementos asocia- 
dos a baja probabilidad de SCA (dolor pleurítico, posicional o punzante) característicamente mostraban un bajo nivel de concordancia entre distintos observadores $^{20}$, limitando el poder predictivo negativo de los datos anamnésticos. Por esto, es necesario incorporar otros elementos en la evaluación inicial de estos pacientes.

El ECG es una potente herramienta clínica en la evaluación del dolor torácico, con un elevado poder predictivo negativo en caso de ser normal, tal y como ha sido confirmado por nuestro estudio y por otros autores ${ }^{21}$. Sin embargo, existe una elevada variabilidad en la interpretación del ECG en el contexto clínico del dolor torácico, la que puede depender de múltiples factores como sesgo por expectativas o entrenamiento del observador. Estas limitaciones pueden subsanarse, al menos parcialmente, con una definición pre-hoc de las alteraciones que deben ser dirigidamente identificadas en el electrocardiograma, tal y como fue realizado en nuestro estudio.

La evaluación integral de estos hallazgos anamnésticos y electrocardiográficos permitió la elaboración de un puntaje diagnóstico que permitió la correcta identificación de los pacientes a los que, finalmente, se les diagnosticó un infarto sin supradesnivel del segmento ST. Estos resultados se comparan favorablemente con otros algoritmos de estratificación de riesgo ${ }^{22}$, destacando su simplicidad en la implementación e interpretación, lo que permite orientar y hacer más efectiva y rápida la toma de decisiones en la evaluación inicial de pacientes con DT. Un estudio que evaluará prospectivamente este algoritmo se encuentra actualmente en curso en nuestra institución.

La evaluación sistemática de elementos clínicos, electrocardiográficos y la determinación de biomarcadores en pacientes seleccionados permitió obtener resultados satisfactorios en cuanto a

\section{REFERENCIAS}

1. Farkouh MF, Smars Pa, Reeder GS, Zinsmeister AR, Evans RN, Meloy TD et al. A clinical trial of a chest-pain observation unit for patients with unstable angina. Chest Pain Evaluation in the diagnóstico y tiempo de evaluación en la UDT. Se confirmó el diagnóstico de SCA en 71\% de los pacientes hospitalizados desde la UDT, comparándose estos resultados favorablemente con la literatura publicada ${ }^{16}$. Es relevante notar que, a diferencia de otras estrategias de UDT publicadas $23-25$, en nuestro centro no se recurrió a técnicas no invasivas (prueba de esfuerzo convencional o con radioisótopos) para la estratificación de pacientes en riesgo intermedio, sin que esto implicase un impacto significativo en la sensibilidad o especificidad de nuestro algoritmo. En otras publicaciones en que se ha analizado el valor de una prueba de esfuerzo complementaria después de 6-12 h, sólo se ha refrendado el diagnóstico clínico inicial ${ }^{26-27}$. Ello avala aún más la implementación de una estrategia fundamentalmente clínica, de menor costo y que evita el eventual riesgo de una prueba de provocación de isquemia en pacientes que consultan por DT. En los pacientes enviados a su domicilio no se observaron eventos clínicos de importancia, aun cuando las características del seguimiento, el hecho que la población provenga de un solo centro y el análisis retrospectivo de los datos pueden limitar la generalización de las conclusiones.

Consideramos que el presente estudio es valioso porque representa una experiencia que incluye a un gran número de pacientes que consultan por DT en nuestro medio, con resultados similares a lo publicado en la literatura internacional. Nuestros hallazgos reafirman el valor de la evaluación clínica y sugieren que la implementación de UDT para el manejo del DT en el SU es factible y útil, aun en lugares sin métodos especializados de detección de isquemia. Esta estrategia permite optimizar el cuidado del paciente, previniendo altas inadecuadas y evitando a la vez hospitalizaciones innecesarias.
Emergency Room (CHEER) Investigators. N Engl J Med 1998; 339: 1882-8.

2. Arós F, Loma-Osorio A. [Diagnosis of unstable angina in the emergency room. The clinical value and limitations of electrocardiography and other tests]. Rev Esp Cardiol 1999; 52 Suppl 1: 39-45. 
3. Lee TH, Rouan GW, Weisberg MC, Brand DA, Acampora D, Stasiulewicz et al. Clinical characteristics and natural history of patients with acute myocardial infarction sent home from the emergency room. Am J Cardiol 1987; 60: 219-24.

4. Storrow AB, Gilber WB. Chest pain centers: diagnosis of acute coronary syndromes. Ann Emerg Med 2000; 35: 449-61.

5. Goodacre SW, Angelini JA, Revill S, Morris F. Clinical predictors of acute coronary syndromes in patients with undifferentiated chest pain. Q J Med 2003; 96: 893-8.

6. Panju AA, Hemmelgarn BR, Guyatt GH, Simel DL. The rational clinical examination. Is this patient having a myocardial infarction? JAMA 1998; 280 : 1256-63.

7. Westerhout CM, Fu Y, Lauer MS, James S, Armstrong PW, Al-Hattab E et al. Short and long term risk stratification in acute coronary syndromes: the added value of quantitative ST-segment depression and multiple biomarkers. J Am Coll Cardiol 2006; 48: 939-47.

8. Januzzi JL, Newby LK, Murphy SA, Pieper K, Antman EM, Morrow DA et al. Predicting a late positive serum troponin in initially troponin-negative patients with non-ST-elevation acute coronary syndrome: clinical predictors and validated risk score results from TIMI IIIB and GUSTO IIA studies. Am Heart 2006; 151: 360-6.

9. Kosuge M, Kimura K, Ishikawa T, Ebina T, Hibi K, Tsukahara K ET al. Combined pronostic utility of ST segment in lead aVR and troponin $\mathrm{T}$ on admission in non-ST-segment elevation acute coronary syndromes. Am J Cardiol 2006; 97 : 334-9.

10. De Araújo Gonçalves P, Ferreira J, Aguiar C, SeabraGomes R. TIMI, PURSUIT, and GRACE risk scores: sustained prognostic value and interaction with revascularization in NSTE-ACS. Eur Heart J 2005; 26: $865-72$.

11. Antman EM, Cohen M, Bernink PJ, McCabe CH, Horacek T, PAPUChIS G ET AL. The TIMI risk score for unstable angina/non-ST elevation MI: A method for prognostication and therapeutic decision making. JAMA 2000; 284: 835-42.

12. Bayón J, Alegría E, Bosh X, Genover X, Cabadés A, IgLesias I ET AL. [Chest pain units. Organization and protocol for the diagnosis of acute coronary syndromes]. Rev Esp Cardiol 2002; 55: 143-54.
13. Lewis WR, Amsterdam EA. Defining the role of chest pain units. J Am Coll Cardiol 2001; 37: 2050-2.

14. Zalenski RJ, Selker HP, Cannon CP, Farin HM, Gibler WB, Goldberg RJ et al. National Heart Attack Alert Program position paper: chest pain centers and programs for the evaluation of acute cardiac ischemia. Ann Emerg Med 2000; 35: 462-71.

15. Pastor Torres LF, Pavon-Jiménez R, Reina Sánchez M. [Chest pain unit: one-year follow-up]. Rev Esp Cardiol 2002; 55: 1021-7.

16. Goodacre S, Nicholl J, DiXon S. Randomized controlled trial and economic evaluation of a chest pain observation unit compared with routine care. BMJ 2004; 328: 254.

17. Bassan R, Gilbler WB. [Chest pain units: state of the art of the management of patients with chest pain in the emergency department]. Rev Esp Cardiol 2001; 54: 1103-9.

18. Gómez MA, Anderson JL, Karagounis LA, Muhlestein JB, Mooers FB et al. An emergency departmentbased protocol for rapidly ruling out myocardial ischemia reduces hospital time and expense: results of a randomized study (ROMIO). J Am Coll Cardiol 1996; 28: 25-33.

19. Kannel WB, Аввотt RD. Incidence and prognosis of unrecognized myocardial infarction. N Engl J Med 1984; 311: 1144-7.

20. HiCKan DH, Sox HC, Sox CH. Systematic bias in recording the history in patients with chest pain. J Chronic Dis 1985; 38: 91-100.

21. Jonsbu J, Rollag A, Aase O. Rapid and correct diagnosis of myocardial infarction: standardized case history and clinical examination provide important information for correct referral to monitored beds. J Intern Med 1991; 229: 143-9.

22. Pozen MW, D'Agostino RB, Selker HP, Sytkowski PA, Hood WB. A predictive instrument to improve coronary-care-unit admission practices in acute ischemic heart disease. A prospective multicenter clinical trial. N Engl J Med 1984; 310: 1273-8.

23. Stein R, Chaitman B, Balady J, Fleq JL, Limacher MC, PINA IL ET aL. Safety and utility of exercise testing in emergency room chest pain centers: An advisory from the Committee on Exercise, Rehabilitation, and Prevention, Council on Clinical Cardiology, American Heart Association. Circulation 2000; 102: 1463-7. 
24. Sanchis J, Bodi V, Llacer A, Nunez J, Ferrero JA, Chorro FJ ET Al. [Value of early exercise stress testing in a chest pain unit protocol]. Rev Esp Cardiol 2002; 55: 1089-92.

25. Conti A, Zanobetti M, Grifoni S, Berni G, Costanzo E, Gallini C ET AL. Implementation of myocardial perfusion imaging in the early triage of patients with suspected acute coronary syndromes. Nucl Med Commun 2003; 24: 1055-60.

26. Martínez-Sellés M, Ortiz J, Estévez A, Anduela J,
De Miguel J, Bueno H. [A new risk score for patients with a normal or non-diagnostic ECG admitted to a chest pain unit]. Rev Esp Cardiol 2005; 58: 782-8.

27. Castillo Moreno Ja, Ramos Martín JL, Molina Laborda E, Egea Beneyto S, Ortega Bernal J. [Usefulness of clinical profiling and exercise testing in the prognostic assessment of patients admitted with chest pain but without high-risk criteria]. Rev Esp Cardiol 2006; 59: 12-9. 